\title{
Equivalence of Ensembles in Quantum Lattice Systems: States
}

\author{
R. LIMA \\ Centre de Physique Théorique, C.N.R.S., Marseille $9^{\mathrm{e}}$, France
}

Received May 19, 1971

\begin{abstract}
The general analysis of the equivalence of ensembles in quantum lattice systems, which was undertaken in paper I of this series, is continued.

The properties of equilibrium states are considered in a variational sense. It is then shown that there exists a canonical as well as a microcanonical variational formulation of equilibrium both of which are equivalent to the grandcanonical formulation.

Equilibrium states are constructed both in the canonical and in the microcanonical formalism by means of suitable limiting procedures.

It is shown, in particular, that the invariant equilibrium states for a given energy and density are those for which the maximum of the mean entropy is reached. The mean entropy thus obtained coincides with the microcanonical entropy.
\end{abstract}

\section{Introduction}

In a previous paper [1], the problem of the equivalence of ensembles in Quantum Lattice Systems was begun. The purpose of this paper is to continue the analysis of equivalence of ensembles in quantum spin systems. In [1] we gave an algebraic formulation of the mathematical framework of quantum spin systems in the three usual ensembles and also some equivalence formulas of the respective thermodynamic functions. This allowed us to show some properties in one ensemble if they are proved in another.

In the present we continue in the same way and we consider the properties of the equilibrium states using a variational principle introduced by Ruelle for the grandcanonical ensemble [2].

We consider a quantum lattice system on $Z^{v}$. We associate with each lattice site $x \in Z^{v}$ a Hilbert space $\mathscr{H}_{x}$ of dimension two, and with each finite region $\Lambda$ in $Z^{v}$ the tensor product

$$
\mathscr{H}(\Lambda)=\bigotimes_{x \in \Lambda} \mathscr{H}_{x} .
$$

If $\Lambda_{1} \subset \Lambda_{2}$ we can identify each bounded operator $A$ on $\mathscr{H}\left(\Lambda_{1}\right)$ with $A \otimes 1_{\Lambda_{2} / \Lambda_{1}}$ on $\mathscr{H}\left(\Lambda_{2}\right)$, where $1_{\Lambda_{2} / \Lambda_{1}}$ is the identity of $\mathscr{H}\left(\Lambda_{2} / \Lambda_{1}\right)$. With this 
convention one defines the algebra of observables by the following

$$
\mathfrak{U}=\bar{\bigcup}_{\Lambda \in Z^{v}} \mathfrak{A}(\Lambda)
$$

where $\mathfrak{U}(\Lambda)$ is the set of bounded operators on $\mathscr{H}(\Lambda)$.

We note that the group $Z^{v}$ of space translations is a subgroup of the automorphism group of $\mathfrak{A}$ and we denote the action of this group by

$$
A \in \mathfrak{U}(\Lambda) \rightarrow \tau_{a} A \in \mathfrak{A}(\Lambda+a), \quad a \in Z^{v} .
$$

We consider interactions, i.e., functions $\Phi$ from the set of finite subsets of $Z^{v}$ to $\mathfrak{A}$ such that

i) $\Phi(X) \in \mathfrak{A}(X), \forall X \subset Z^{v}$,

ii) $\Phi(X)$ is hermitian,

iii) $\Phi(X+a)=\tau_{a} \Phi(X), \quad \forall a \in Z^{v}$,

iv) $\|\Phi\|=\sum_{X \ni 0} \frac{\|\Phi(X)\|}{N(X)}<+\infty$

where the last sum extends over all finite subsets of $Z^{v}$ containing 0 and $N(X)$ is the number of points of $X$.

We denote by $\mathfrak{B}$ the set of such interactions.

We consider a system of particles on the finite set $\Lambda$ and the energy operator $U_{\Phi}(\Lambda) \in \mathfrak{A}(\Lambda)$ corresponding to the interaction $\Phi$ defined by

$$
U_{\Phi}(\Lambda)=\sum_{X \subset A} \Phi(X) \text {. }
$$

We also introduce the "interaction energy" at the origin by

$$
A_{\Phi}=\sum_{X \ni 0} \frac{\Phi(X)}{N(X)}
$$

Further we denote by $\left\{e_{0}^{(x)}, e_{1}^{(x)}\right\}$ an orthonormal basis of $\mathscr{H}_{x}$, for each $x \in Z^{v}$.

Now for each finite region $\Lambda \subset Z^{v}$, we define a configuration $|X\rangle$ which is at once a subset $\left\{x_{1}, \ldots, x_{k}\right\}$ of $\Lambda$ and an element of $\mathscr{H}(\Lambda)$ defined by

$$
|X\rangle=\bigotimes_{x \in \Lambda} e_{\delta(x)}^{(x)}
$$

where $\delta(x)=1$ if $x \in\left\{x_{1}, \ldots, x_{k}\right\}$ and 0 if not.

If $\Lambda_{1} \subset \Lambda_{2}$ we can identify every configuration $|X\rangle$ of $\mathscr{H}\left(\Lambda_{1}\right)$ with $|X\rangle \otimes \emptyset_{\Lambda_{2} / \Lambda_{1}}$ of $\mathfrak{A}\left(\Lambda_{2}\right)$, where $\emptyset_{\Lambda_{2} / \Lambda_{1}}$ is the vacuous subset of $\Lambda_{2} / \Lambda_{1}$. Clearly the set of all configurations of $\Lambda$ is an orthonormal basis of $\mathscr{H}(\Lambda)$.

We define projectors $P^{N}(\Lambda) \in \mathfrak{A}(\Lambda) ; 0 \leqq N \leqq N(\Lambda)$, by

$$
\begin{aligned}
P^{N}(\Lambda)|X\rangle & =|X\rangle \\
& =0 \quad \text { if } N(X)=N \\
& \text { if not } .
\end{aligned}
$$


Now we give a review of definitions in the three ensembles which be used in the following.

In the microcanonical formalism variables are the energy per unit of volume $e$, and the density $n, 0 \leqq n \leqq 1$.

For each finite region $\Lambda$ of $Z^{v}$ and interaction $\Phi \in \mathfrak{B}$, we can define the microcanonical partition function by

$$
\Omega_{\Lambda}^{\Phi}(E, N)=\operatorname{Tr}_{\mathscr{H}(\Lambda)}\left\{P^{N}(\Lambda) \sum_{\lambda_{i}(\Phi, \Lambda) \leqq E} E_{\lambda_{i}(\Phi, \Lambda)}\right\}
$$

where $E=e \cdot N(\Lambda), N$ is an integer such that $0 \leqq N \leqq N(\Lambda)$ and $\left\{\lambda_{i}(\Phi, \Lambda)\right\}_{i \geqq 0}$ is the set of eigenvalues of $U_{\Phi}(\Lambda)$ repeated according to multiplicity and $\left\{E_{\lambda_{i}(\Phi, \Lambda)}\right\}_{i \geqq 0}$ is the corresponding set of spectral projectors.

We define the microcanonical thermodynamic function, actually the entropy, by

$$
s^{\Phi}(e, n)=\lim _{\substack{\Lambda \rightarrow \infty \\ \frac{N}{N(\Lambda)} \rightarrow n}} \frac{1}{N(\Lambda)} \log \Omega_{\Lambda}^{\Phi}(e \cdot N(\Lambda), N) .
$$

In the canonical formalism variables are the density $n$ and the inverse temperature $\beta$.

For each finite region $\Lambda \subset Z^{v}$ and interaction $\Phi \in \mathfrak{B}$, we can also define a canonical partition function

$$
\Xi_{\Lambda}^{\Phi}(N, \beta)=\operatorname{Tr}_{\mathscr{H}(\Lambda)}\left\{P^{N}(\Lambda) \exp \left(-\beta U_{\Phi}(\Lambda)\right)\right\}
$$

where $N$ is an integer such that $0 \leqq N \leqq N(\Lambda)$.

We define the canonical thermodynamic function, actually the free energy by

$$
f^{\Phi}(n, \beta)=-\beta^{-1} \lim _{\substack{\Lambda \rightarrow \infty \\ \frac{N}{N(\Lambda)} \rightarrow n}} \frac{1}{N(\Lambda)} \log \Xi_{\Lambda}^{\Phi}(N, \beta) .
$$

In the grandcanonical formalism variables are the inverse temperature $\beta$ and the chemical potential $\mu$.

For each finite region $\Delta \subset Z^{v}$ and interaction $\Phi \in \mathfrak{B}$, we can also define a grandcanonical partition function

$$
Z_{\Lambda}^{\Phi}(\beta, \mu)=\operatorname{Tr}_{\mathscr{H}(\Lambda)}\left\{\exp \left(\beta \mu \mathcal{N}(\Lambda)-\beta U_{\Phi}(\Lambda)\right)\right\}
$$

where $\mathscr{N}(\Lambda) \in \mathfrak{U}(\Lambda)$ is defined by

$$
\forall X \subset \Lambda \mathscr{N}(\Lambda)|X\rangle=N(X)|X\rangle .
$$

We define the grandcanonical thermodynamic function, actually the pressure, by

$$
p^{\Phi}(\beta, \mu)=\beta^{-1} \lim _{\Lambda \rightarrow \infty} \frac{1}{N(\Lambda)} \log Z_{\Lambda}^{\Phi}(\beta, \mu)
$$


Let $E$ be the set of the normalized positive linear functionals over $\mathfrak{A}$, i.e. the set of the states and $E \cap L_{Z^{v}}^{\perp}$ the subset of $E$ of the translationnally invariant states, i.e. such that

$$
\varrho \in E \quad \varrho\left(\tau_{x} A\right)=\varrho(A) \quad \forall A \in \mathfrak{A} \quad \text { and } \quad \forall x \in Z^{v} .
$$

If $\varrho \in E$, the restriction of $\varrho$ to any $\mathfrak{A}(\Lambda)$, the state defines a positive operator $\varrho_{A}$ on $\mathscr{H}(\Lambda)$ such that

$$
\operatorname{Tr}_{\mathscr{H}(A)}\left\{\varrho_{A}\right\}=1 \text { and } \operatorname{Tr}_{\mathscr{H}(\Lambda)}\left\{\varrho_{\Lambda} A\right\}=\varrho(A)
$$

for $A \in \mathfrak{A}(\Lambda)$.

We refer to [3] or [4] for the properties of the mean entropy of an invariant state, defined by

$$
S(\varrho)=\lim _{\Lambda \rightarrow \infty}-\frac{1}{N(\Lambda)} \operatorname{Tr}_{\mathscr{H}(\Lambda)}\left\{\varrho_{\Lambda} \log \varrho_{\Lambda}\right\}
$$

where the limit $\Lambda \rightarrow \infty$ is the sense of the parallelepipeds. The mean entropy is a non-negative affine upper semi-continuous function on $E \cap L_{Z^{v}}^{\perp}$.

As in [1] we consider only the interactions which commute with all the $P^{N}(\Lambda)$ for each $\Lambda$.

\section{Variational Properties}

With the foregoing definitions the Variational Property (Theorem IV of [3] and Theorem III of [4]) has the following form:

Theorem 1. If $\Phi \in \mathfrak{B}$ then we have for $\beta>0$ and $\mu \in \mathfrak{R}$

$$
\beta p^{\Phi}(\beta, \mu)=\sup _{\varrho \in E \cap L_{Z^{v}}}\left\{S(\varrho)-\beta \varrho\left(A_{\Phi}\right)+\beta \mu \varrho\left(\mathscr{N}_{0}\right)\right\}
$$

and

$$
S(\varrho)=\inf _{\Phi \in \mathfrak{B}}\left\{\beta p^{\Phi}(\beta, \mu)+\beta \varrho\left(A_{\Phi}\right)-\beta \mu \varrho\left(\mathscr{N}_{0}\right)\right\} \quad \text { for } \quad \varrho \in E \cap L_{Z^{v}}^{\perp} .
$$

Once one notes that this Theorem is identical to that of [3] because

$$
\beta p^{\Phi}(\beta, \mu)=P\left(\Phi^{\prime}\right)
$$

where $P($.$) is the pression as defined in [2] and \Phi^{\prime}$ is connected with $\Phi$ by the following:

$$
\begin{array}{lll}
\Phi^{\prime}(\{x\})=-\beta \mu \mathcal{N}_{x}+\beta \Phi(\{x\}) & x \in \Lambda & \\
\Phi^{\prime}(X)=\beta \Phi(X) & X \subset \Lambda \text { and } & N(X) \neq 1 .
\end{array}
$$

The aim of this section is to describe this variational property in the canonical and microcanonical ensembles. 
First, for canonical ensemble, it was shown in [1] that thermodynamic functions of grand canonical and canonical ensembles are related by the following

$$
f^{\Phi}(n, \beta)=\sup _{\mu \in \mathfrak{R}}\left\{\mu n-p^{\Phi}(\beta, \mu)\right\}
$$

for $\Phi \in \mathfrak{B}, 0<n<1, \beta>0$ and $\mu \in \mathfrak{R}$.

Then, we can show the following.

Theorem 2. If $\Phi \in \mathfrak{B}$, then we have for $\beta>0$ and $0<n<1$

(I) $-\beta f^{\Phi}(n, \beta)=\sup _{\substack{\varrho \in E \cap L_{Z^{v}} \\ \varrho\left(N_{0}\right)=n}}\left\{S(\varrho)-\beta \varrho\left(A_{\Phi}\right)\right\}$.

\section{Furthermore}

(II) $S(\varrho)=\inf _{\Phi \in \mathfrak{B}}\left\{-\beta f^{\Phi}\left(\varrho\left(\mathscr{N}_{0}\right), \beta\right)-\beta \varrho\left(A_{\Phi}\right)\right\} \quad$ for $\varrho \in E \cap L_{Z^{v}}^{\perp}$.

We put

$$
-\beta \hat{f}^{\Phi}(n, \beta)=\sup _{\substack{\varrho \in E \cap L_{Z^{\nu}} \\ \varrho\left(\mathcal{N}_{0}\right)=n}}\left\{S(\varrho)-\beta \varrho\left(A_{\Phi}\right)\right\}
$$

and we begin by proving that

$$
-\beta f^{\Phi}(n, \beta) \geqq-\beta \hat{f}^{\Phi}(n, \beta) .
$$

By Theorem 1, we have, for all $\varrho \in E \cap L_{Z^{v}}^{\perp}$ such that $\varrho\left(\mathscr{N}_{0}\right)=n$ and all $\mu \in \mathfrak{R}$

and by (2.3)

$$
\beta p^{\Phi}(\beta, \mu) \geqq S(\varrho)-\beta \varrho\left(A_{\Phi}\right)+\beta \mu n
$$

$$
-\beta f^{\Phi}(n, \beta)=\beta \inf _{\mu \in \mathfrak{R}}\left\{p^{\Phi}(\beta, \mu)-\mu n\right\} \geqq-\beta \hat{f}^{\Phi}(n, \beta) .
$$

Now $\hat{f}^{\Phi}(n \cdot \beta)$ is a convex function of $n$ because for $0 \leqq \lambda, n_{1}, n_{2} \leqq 1$

$$
\begin{aligned}
& -\beta \hat{f}^{\Phi}\left(\lambda n_{1}+(1-\lambda) n_{2}, \beta\right) \\
& \geqq \sup _{\substack{\varrho_{1}, \varrho_{2} \in E \cap L_{Z_{Z}}^{1} \\
\varrho_{1}\left(\mathcal{N}_{0}\right)=n_{1} \\
\varrho_{2}\left(\mathcal{N}_{0}\right)=n_{2}}}\left\{S\left(\lambda \varrho_{1}+(1-\lambda) \varrho_{2}\right)-\beta \lambda \varrho_{1}\left(A_{\Phi}\right)-\beta(1-\lambda) \varrho_{2}\left(A_{\Phi}\right)\right\} \\
& =\sup _{\substack{\varrho_{1}, \varrho_{2} \in E \cap E_{L_{Z}}^{1} \\
\varrho_{1}\left(\mathcal{N}_{0}\right)=n_{1} \\
\varrho_{1}\left(\mathcal{N}_{0}\right)=n_{2}}}\left\{\lambda\left[S\left(\varrho_{1}\right)-\beta \varrho_{1}\left(A_{\Phi}\right)\right]+(1-\lambda)\left[S\left(\varrho_{2}\right)-\beta \varrho_{2}\left(A_{\Phi}\right)\right]\right\} \\
& =-\lambda \beta \hat{f}^{\Phi}\left(n_{1}, \beta\right)-(1-\lambda) \beta \hat{f}^{\Phi}\left(n_{2}, \beta\right) .
\end{aligned}
$$

So for $\beta$ and $\Phi$ fixed

$$
\begin{gathered}
\forall n \in] 0,1\left[\quad \exists \mu_{n} \in \Re \quad \forall l \in[0,1]\right. \\
\hat{f}^{\Phi}(l, \beta) \geqq \hat{f}^{\Phi}(n, \beta)-\mu_{n}(n-l)
\end{gathered}
$$


and it is shown in [1] that

Furthermore

$$
f^{\Phi}(n, \beta)=\mu_{n} n-p^{\Phi}\left(\beta, \mu_{n}\right)
$$

$$
\forall \varepsilon>0 \quad \exists \dot{\varrho} \in E \cap L_{Z^{v}}^{\perp}
$$

such that

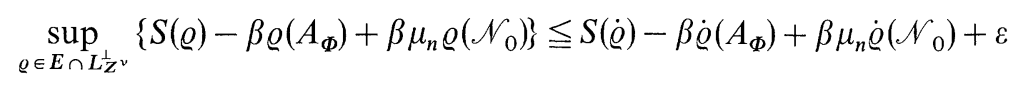

and put $\varrho\left(\mathcal{N}_{0}\right)=l$.

Therefore

$$
\begin{aligned}
\beta \hat{f}^{\Phi}(n, \beta) & =\beta \mu_{n} n-\beta p^{\Phi}\left(\beta, \mu_{n}\right) \\
& =\beta \mu_{n} n-\sup _{\varrho \in E \cap L_{Z^{v}}}\left\{S(\varrho)-\beta \varrho\left(A_{\Phi}\right)+\beta \mu_{n} \varrho\left(\mathscr{N}_{0}\right)\right\} \\
& \geqq \beta \mu_{n} n-S(\varrho)+\beta \varrho\left(A_{\Phi}\right)-\beta \mu_{n} \varrho\left(\mathscr{N}_{0}\right)-\varepsilon \\
& \geqq \beta \mu_{n}(n-l)-\sup _{\substack{\varrho \in E \cap L_{Z^{v}} \\
\varrho\left(\mathcal{V}_{0}\right)=l}}\left\{S(\varrho)-\beta \varrho\left(A_{\Phi}\right)\right\}-\varepsilon \\
& =\beta \mu_{n}(n-l)+\beta \hat{f}^{\Phi}(l, \beta)-\varepsilon \\
& \geqq \beta \hat{f}^{\Phi}(n, \beta)-\varepsilon
\end{aligned}
$$

where we have used (2.11) the Theorem 1 and the convexity property (2.10). Combining (2.8) and (2.12) part (I) follows. Part (II) follows from part (I) by analogy with 5 of Theorem 2 of [4]. We remark that, also with results of [1] and the fact that the function

$$
\sup _{\varrho \in E \cap L_{Z^{v}}}\left\{S(\varrho)-\beta \varrho\left(A_{\Phi}\right)+\beta \mu \varrho\left(\mathscr{N}_{0}\right)\right\}
$$

is convex in $\mu$, we show in the same way that Theorem 1 follows from Theorem 2.

We recall that $A_{\Phi}$ is interpretated as the mean energy per site associated with an interaction $\Phi \in \mathfrak{B}$ and if $\varrho$ is an invariant state $\varrho\left(A_{\Phi}\right)$ may be considered as the expectation value of the energy per site for the interaction $\Phi$ in the state $\varrho$.

The following Theorem is thus an easy physical interpretation completed in the following section.

Theorem 3. If $\Phi \in \mathfrak{B}$ we have for $|e| \leqq\|\Phi\|$ and $0<n<1$

(I) $S^{\Phi}(e, n)=\sup _{\substack{\varrho \in E \cap L_{Z^{\prime}} \\ \varrho\left(\mathscr{N}_{0}\right)=n ; \varrho\left(A_{\Phi}\right)=e}} S(\varrho)$.

\section{Furthermore}

(II) $S(\varrho)=\inf _{\Phi \in \mathfrak{B}} s^{\Phi}\left(\varrho\left(A_{\Phi}\right), \varrho\left(\mathcal{N}_{0}\right)\right) \quad$ for $\quad \varrho \in E \cap L_{Z^{v}}^{\perp}$ 
The proof of this Theorem is similar to the previous one. We remark only that for each $n$ the function

$$
\sup _{\substack{\left.\varrho \in E \cap L L_{Z^{\prime} v} \\ \mathcal{W}_{0}\right)=n ; \varrho\left(A_{\Phi}\right)=e}} S(\varrho)
$$

is concave in $e$ by the following

$$
\begin{aligned}
& \sup _{\substack{\varrho \in E \cap L_{Z^{v}} \\
\varrho\left(\mathcal{N}_{0}\right)=n ; \varrho\left(A_{\Phi}\right)=\lambda e_{1}+(1-\lambda) e_{2}}} S(\varrho) \geqq \sup _{\substack{\varrho_{1}, \varrho_{2} \in E \cap L_{L^{v}} \\
\varrho_{1}\left(\mathcal{N}_{0}\right)=\varrho_{2}\left(\mathcal{L}_{0}\right)=n \\
\varrho_{1}\left(A_{\Phi}\right)=e_{1} ; \varrho_{2}\left(A_{\Phi}\right)=e_{2}}} S\left(\lambda \varrho_{1}+(1-\lambda) \varrho_{2}\right) \\
& =\lambda \sup _{\substack{\varrho_{1} \in E \cap L_{Z^{v}} \\
\varrho_{1}\left(N_{0}\right)=n ; \varrho_{1}\left(A_{\Phi}\right)=e_{1}}} S\left(\varrho_{1}\right)+(1-\lambda) \sup _{\substack{\varrho_{2} \in E \cap L_{Z_{Z}} \\
\varrho_{2}\left(N_{0}\right)=n ; \varrho_{2}\left(A_{\Phi}\right)=e_{2}}} S\left(\varrho_{2}\right)
\end{aligned}
$$

where $0 \leqq \lambda \leqq 1$.

And so, we can use results of [1] and Theorem 2 for proving the present Theorem.

We remark also that the function

$$
\left.\sup _{\substack{\varrho \in E \cap L_{Z^{v}} \\ \varrho\left(\mathcal{N}_{0}\right)=n}}\left\{S(\varrho)-\beta \varrho A_{\Phi}\right)\right\}
$$

is convex in $\beta$ and using also results of [1] this proves that part I of Theorem 2 follows from part I of Theorem 3 .

Part II follows by analogy with Theorem 2 and 1 and continuity of $s^{\Phi}(e, n)$ in $(e, n)$.

\section{Equilibrium States}

In Quantum Statistical Mechanics the ensemble averages are given by a positive operator of trace class. If $\Lambda^{\prime}$ is a subsystem of the lattice confined to $\Lambda$, for all $A \in \mathfrak{A}\left(\Lambda^{\prime}\right)$ we define a ensemble average of $A$ by

$$
\varrho_{\Phi, \Lambda}(A)=\operatorname{Tr}\left\{T_{\Lambda}^{\Phi}\right\}^{-1} \operatorname{Tr}\left\{T_{\Lambda}^{\Phi} A\right\}
$$

where $T_{A}^{\Phi}$ is the ensemble unnormalized density matrix, meaning in the grand canonical ensemble:

$$
T_{\Lambda}^{\Phi}=\exp \left\{\beta \mu \mathscr{N}(\Lambda)-\beta U_{\Phi}(\Lambda)\right\}
$$

in the canonical ensemble:

$$
T_{\Lambda}^{\Phi}=P^{N}(\Lambda) \exp \left\{-\beta U_{\Phi}(\Lambda)\right\}
$$

in the micro canonical ensemble:

$$
T_{\Lambda}^{\Phi}=P^{N}(\Lambda) \sum_{\lambda_{i} \in E_{\Lambda}^{A}} E_{\lambda_{i}(\Phi, \Lambda)}
$$

where $E_{\Lambda}^{\Delta}$ is the interval $[e N(\Lambda), N(\Lambda)(e+\Delta)]$ for $\Delta \in \mathfrak{R}^{+}$. 
As in the grand canonical ensemble [3] and [4] we will prove the existence of invariant states reaching the maximum of formulas of Theorems 2 and 3 and for a "large" set of interactions they may be obtained as thermodynamical limit of the averages before ${ }^{1}$.

We recall that in [3] is proved that for each $(\Phi, \beta, \mu)$ we can associate at least one invariant state $\varrho_{\Phi, \beta, \mu}$, such that

$$
\begin{aligned}
\beta p^{\Phi}(\beta, \mu) & =S\left(\varrho_{\Phi, \beta, \mu}\right)-\varrho_{\Phi, \beta, \mu}\left(\beta A_{\Phi}-\beta \mu\left(\mathscr{N}_{0}\right)\right. \\
& =\sup _{\varrho \in E \cap L_{Z^{\prime}}^{1}}\left\{S(\varrho)-\beta \varrho\left(A_{\Phi}\right)+\beta \mu \varrho\left(\mathscr{N}_{0}\right)\right\} .
\end{aligned}
$$

It is proved in [4] that each tangent functional $\alpha_{\Phi, \beta, \mu}($.$) to the graph of$ $p^{\Phi}(\beta, \mu)$ determines an (invariant) equilibrium state that verifies (3.1). More precisely the situation is the following:

Let $T_{1} \subset \mathfrak{B} \times \mathfrak{R}^{+} \times \mathfrak{R}$ be the set of $(\Phi, \beta, \mu) \in \mathfrak{B} \times \mathfrak{R}^{+} \times \mathfrak{R}$ such that the graph of $p$ has a unique tangent functional at this point, i.e.

$$
\begin{aligned}
p^{\Phi+\Phi_{1}}\left(\beta, \mu+\mu_{1}\right) \geqq p^{\Phi}(\beta, \mu)-\alpha_{\Phi, \beta, \mu}\left(\Phi_{1}, \mu_{1}\right) & \forall \Phi_{1} \in \mathfrak{B}, \\
& \forall \mu_{1} \in \mathfrak{R}
\end{aligned}
$$

determines a unique $\alpha_{\Phi, \beta, \mu}(.,.) \in(\mathfrak{B} \times \mathfrak{R})^{\prime}$ for $(\Phi, \beta, \mu) \in T_{1}$.

Now $\alpha_{\Phi, \beta, \mu}(., 1)$ determines a state $\varrho_{\Phi, \beta, \mu} \in E \cap L_{Z^{v}}^{\perp}$ through the relation

$$
\alpha_{\Phi, \beta, \mu}(\Psi, 1)=-\varrho_{\Phi, \beta, \mu}\left(\mathscr{N}_{0}\right)+\varrho_{\Phi, \beta, \mu}\left(A_{\Psi}\right) \quad \forall \Psi \in \mathfrak{B} .
$$

The state $\varrho_{\Phi, \beta, \mu}$ gives the maximum in (3.1) and this maximum is unique only if $(\Phi, \beta, \mu) \in T_{1}$.

Now we can investigate some properties of these equilibrium states using the Theorems 2 and 3.

We begin with following

Theorem 4. Let $\Phi \in \mathfrak{B}$. For each $\mu \in \mathfrak{R}$

i) we can define a non empty set $\hat{n}_{\mu} \subset[0,1]$ of the $n_{\mu}$ such that

$$
p^{\Phi}(\beta, \mu)=\mu n_{\mu}-f^{\Phi}\left(n_{\mu}, \beta\right),
$$

ii) there exists at least one (invariant) equilibrium state $\varrho_{\Phi, \beta, \mu}$ that verifies (3.1) and

$$
\varrho_{\Phi, \beta, \mu}\left(\mathscr{N}_{0}\right) \in \hat{n}_{\mu},
$$

$\varrho_{\Phi, \beta, \mu}$ is unique if and only if $(\Phi, \mu, \beta) \in T_{1}$,

${ }^{1}$ In the microcanonical case we are interested in the case $\Delta \rightarrow 0$. Unfortunately it appears that then, in the Quantum Spin System, the limit $\Lambda \rightarrow \infty$ is connected to the size of convergence $\Delta \rightarrow 0$.

14 Commun. math Phys, Vol 24 
iii) each of these equilibrium states reaches the maximum of Theorem 2

$$
-\beta f^{\Phi}\left(n_{\mu}, \beta\right)=S\left(\varrho_{\Phi, \beta, \mu}\right)-\beta \varrho_{\Phi, \beta, \mu}\left(A_{\Phi}\right)
$$

where $n_{\mu}=\varrho_{\Phi, \beta, \mu}\left(\mathscr{N}_{0}\right)$

and conversely, for each $n \in] 0,1[$.

iv) We can define a non empty set $\hat{\mu}_{n} \subset \mathfrak{R}$ of the $\mu_{n}$ such that

$$
f^{\Phi}(n, \beta)=\mu_{n} n-p^{\Phi}(\beta, \mu) .
$$

v) There exists at least one state $\varrho_{\Phi, n, \beta} \in E \cap L_{Z}^{\perp v}$ such that

$$
\varrho_{\Phi, n, \beta}\left(\mathcal{N}_{0}\right)=n \text { and }-\beta f^{\Phi}(n, \beta)=S\left(\varrho_{\Phi, n, \beta}\right)-\beta \varrho_{\Phi, n, \beta}\left(A_{\Phi}\right) \text {. }
$$

vi) For each of these states there exists $\mu_{n} \in \hat{\mu}_{n}$ such that

$$
\beta p^{\Phi}\left(\beta, \mu_{n}\right)=S\left(\varrho_{\Phi, n, \beta}\right)-\beta \varrho_{\Phi, n, \beta}\left(A_{\Phi}\right)+\beta \mu_{n} \varrho_{\Phi, n, \beta}\left(\mathscr{N}_{0}\right) .
$$

We note $\left.T_{2} \subset \mathfrak{B} \times\right] 0,1\left[\times \mathfrak{R}^{+}\right.$the set $(\Phi, n, \beta)$ such that $\varrho_{\Phi, n, \beta}$ is unique.

Proof. Statements i) and iv) together are proved in [1]. The beginning of this section gives references to the proof of parts of ii).

Now we recall that the set $E \cap L_{Z^{v}}^{\perp}$ of invariant states is weak-*-compact in the set of all states. Then the set of the states such that

$$
\varrho \in E \cap L_{Z^{v}}^{\perp} \text { and } \varrho\left(\mathscr{N}_{0}\right)=n \text { for some } n \in[0,1]
$$

is a closed subset of $E \cap L_{Z^{\nu}}^{\perp}$, because the function $\mathscr{N}(\varrho)=\varrho\left(\mathscr{N}_{0}\right)$ is continuous on $E \cap L_{Z^{v}}^{\perp}$, and therefore weak-*-compact. This proves v) because the function $S(\varrho)-\beta \varrho\left(A_{\Phi}\right)$ is upper-semi-continuous on $E \cap L_{Z^{v}}^{\perp}$. The last inequality of (2.12) proves vi) by the following:

$$
\begin{aligned}
\sup _{\substack{\varrho \in E \cap L_{Z_{v}} \\
\varrho\left(N_{0}\right)=n}}\left\{S(\varrho)-\beta \varrho\left(A_{\Phi}\right)\right\}+\beta \mu_{n} n & =-\beta f^{\Phi}(n, \beta)+\beta \mu_{n} n \\
& \geqq \beta f^{\Phi}(l, \beta)+\beta \mu_{n} l \\
& =\sup _{\substack{\varrho \in E \cap L_{Z^{v}} \\
\varrho\left(N_{0}\right)=l}}\left\{S(\varrho)-\beta \varrho\left(A_{\Phi}\right)\right\}+\beta \mu_{n} l
\end{aligned}
$$

where $\mu_{n}$, as in (2.12) is an element of $\hat{\mu}_{n}$ verifying iv).

Now (3.3) is true for all $l \in[0,1]$, therefore using v) we have

$$
\begin{aligned}
S\left(\varrho_{\Phi, n, \beta}\right)- & \beta \varrho_{\Phi, n \beta}\left(A_{\Phi}\right)+\beta \mu_{n} \varrho_{\Phi, n, \beta}\left(\mathcal{N}_{0}\right) \\
& =\sup _{l \in[0,1]} \sup _{\substack{\varrho \in E \cap L_{Z^{v}} \\
\varrho\left(\mathcal{N}_{0}\right)=l}}\left\{S(\varrho)-\beta \varrho\left(A_{\Phi}\right)+\beta \mu_{n} \varrho\left(\mathcal{N}_{0}\right)\right\} \\
& =\beta p^{\Phi}\left(\beta, \mu_{n}\right) .
\end{aligned}
$$

To prove statement iii) we note that using (3.1) we have

$$
\sup _{\substack{\varrho \in E \cap L_{L^{\prime}} \\ \varrho\left(\mathcal{N}_{0}\right)=\varrho \Phi, \beta, \mu}}\left\{S(\varrho)-\beta \varrho\left(A_{\Phi}\right)\right\}=S\left(\varrho_{\Phi, \beta, \mu}\right)-\beta \varrho_{\Phi, \beta, \mu}\left(A_{\Phi}\right) .
$$


The remaining part of statement ii), i.e.

$$
\varrho_{\Phi, \beta, \mu}\left(\mathscr{N}_{0}\right) \in \hat{n}_{\mu}
$$

follows now by iii) and i).

We remark that if $(\Phi, \beta, \mu) \in T_{1}$ and $\left(\Phi, n_{\mu}, \beta\right) \in T_{2}$ therefore by the previous Theorem

and

$$
\varrho_{\Phi, \beta, \mu}=\varrho_{\Phi, n_{\mu}, \beta}
$$

$$
\varrho_{\Phi, \beta, \mu}\left(\mathscr{N}_{0}\right)=n_{\mu} .
$$

As in the grand canonical ensemble we can compute the equilibrium states associated to each $(\Phi, n, \beta)$ using the tangent planes to the thermodynamics function.

We define:

$$
\alpha_{\Phi} \frac{N}{N(\Lambda)}, \beta(\Psi)=\frac{1}{N(\Lambda)} Z^{\Phi}(N, \beta)^{-1} \operatorname{Tr}_{\mathscr{H}(\Lambda)}\left\{P^{N}(\Lambda) e^{-\beta U_{\Phi}(\Lambda)} U_{\Psi}(\Lambda)\right\}
$$

for $\Phi \in \mathfrak{B}$ and $0 \leqq N \leqq N(\Lambda), \Lambda$ (finite) $\subset Z^{v}$ and we extend this function to all the values of the density parameter $0 \leqq n \leqq 1$ by linearity.

The following Theorem gives information concerning the equilibrium states in the canonical ensemble, i.e. for $\Phi, n, \beta$ fixed.

Theorem 5. Let $T_{2} \subset \mathfrak{B} \times[0,1] \times \mathfrak{R}^{+}$be the set of $(\Phi, n, \beta)$ such that the graph of $f$ has a unique tangent functional in the dual of $\mathfrak{B}$ at $\Phi$ then for $(\Phi, n, \beta) \in T_{2}$ the equilibrium state $\varrho_{\Phi, n, \beta}$ is determined by the tangent functional $\alpha_{\Phi, n, \beta}$ and is ergodic. Further we have for $(\Phi, n, \beta) \in T_{2}$ the relation

$$
\alpha_{\Phi, n, \beta}(\Psi)=\varrho_{\Phi, n, \beta}\left(A_{\Psi}\right)=\lim _{\Lambda \rightarrow \infty} \alpha_{\Phi, n, \beta, \Lambda}(\Psi)
$$

where he limit $\Lambda \rightarrow \infty$ is in the sense of van Hove.

Furthermore, each tangent functional to the graph of $f$ at $(\Phi, n, \beta)$ determines a state $\varrho_{\Phi, n, \beta} \in E \cap L_{Z^{v}}^{\perp}$ through the relation

$$
\alpha_{\Phi, n, \beta}(\Psi)=\varrho_{\Phi, n, \beta}\left(A_{\Psi}\right) .
$$

The proof of the first statement of this Theorem is identical to [2] or [3] once one notes that for finite $\Lambda$ and each $(n, \beta)$ the function $\Phi \rightarrow f_{\Lambda}^{\Phi}(n, \beta)$ has a unique tangent plane and because the linearity of $f_{\Lambda}^{\Phi}(n, \beta)$ in $n$ for $N \leqq n . N(\Lambda) \leqq N+1$ it is $\alpha_{\Phi, n, \beta, \Lambda}$.

The remaining part is identical to part 2 of Theorem 3 of [4].

Clearly $T_{2}$ coincides with the set defined as in Theorem 4 .

The following two Theorems give information about the equilibrium states using the microcanonical formalism. 
Theorem 6. Let $\Phi \in \mathfrak{B}$ and $n \in[0,1]$. For each $\beta>0$

i) we can define a non empty set $\hat{e}_{\beta} \subset E_{\Phi}=[-\|\Phi\|$, $\|\Phi\|]$ of the $e_{\beta}$ such that:

$$
s^{\Phi}\left(e_{\beta}, n\right)=\beta e_{\beta}-\beta f^{\Phi}(n, \beta) .
$$

ii) There exists at least (invariant) equilibrium state $\varrho_{\Phi, n, \beta}$ that verifies the maximum of the canonical variational property of Theorem 2 and

$$
\varrho_{\Phi, n, \beta}\left(A_{\Phi}\right) \in \hat{e}_{\beta} .
$$

iii) Each of this equilibrium state reaches the maximum of Theorem 3, i.e.

where

$$
s^{\Phi}\left(e_{\beta}, n\right)=S\left(\varrho_{\Phi, n, \beta}\right)
$$

and conversely, for each $e \in E_{\Phi}$.

$$
e_{\beta}=\varrho_{\Phi, n, \beta}\left(A_{\Phi}\right)
$$

iv) We can define a non empty set $\hat{\beta}_{e} \subset \mathfrak{R}^{+}$of the $\beta_{e}$ such that:

$$
-\beta_{e} f^{\Phi}\left(n, \beta_{e}\right)=s^{\Phi}(e, n)-\beta_{e} e .
$$

v) There exists at least one state $\varrho_{\Phi, e, n} \in E \cap L_{Z^{v}}^{\perp}$ such that

and

$$
\varrho_{\Phi, e, n}\left(\mathscr{N}_{0}\right)=n ; \quad \varrho_{\Phi, e, n}\left(A_{\Phi}\right)=e
$$

$$
S^{\Phi}(e, n)=S\left(\varrho_{\Phi, e, n}\right) .
$$

vi) For each of these states there exists $\beta_{e} \in \hat{\beta}_{e}$ such that

$$
-\beta_{e} f^{\Phi}\left(n, \beta_{e}\right)=S\left(\varrho_{\Phi, e, n}\right)-\beta \varrho_{\Phi, e, n}\left(A_{\Phi}\right) .
$$

We denote $T_{3} \subset \mathfrak{B} \times E_{\Phi} \times[0,1]$ the set of the $(\Phi, e, n)$ such that $\varrho_{\Phi, e, n}$ is unique.

Proof. Using the equivalence of thermodynamical functions proved in [1] and Theorem 2 and 3 the proof of the Theorem is identical to that of Theorem 4. Once one notes that the function $A_{\Phi}(\varrho)=\varrho\left(A_{\Phi}\right)$ is also weak $*$-continuous and so the set $\left\{\varrho \in E \cap L_{Z^{v}}^{\perp}, \varrho\left(\mathscr{N}_{0}\right)=n\right.$ and $\left.\varrho\left(A_{\Phi}\right)=e\right\}$ is weak *-compact in $E \cap L_{Z^{*}}^{\perp}$.

Like in the other ensembles we can also in the microcanonical ensemble to construct equilibrium states using the finite volume thermodynamic functions.

Let $\Phi \in \mathfrak{B}, \Lambda \in Z^{v}, \Lambda$ finite and $e \in E_{\Phi}, 0 \leqq N \leqq N(\Lambda)$ we define for a given $\Delta \in \mathfrak{R}^{+}$:

$$
\alpha_{\Phi, e, \frac{N}{N(\Lambda)}, \Lambda}^{\Delta}(\Psi)=\frac{1}{N(\Lambda)} \frac{\operatorname{Tr}_{\mathscr{H}(\Lambda)}\left\{P^{N}(\Lambda) \sum_{\lambda_{i} \in E_{\Lambda}^{A}} E_{\lambda_{i}(\Phi, \Lambda)} U_{\Psi}(\Lambda)\right\}}{\operatorname{Tr}_{\mathscr{H}(\Lambda)}\left\{P^{N}(\Lambda) \sum_{\lambda_{i} \in E_{\Lambda}^{\Lambda}} E_{\lambda_{i}(\Phi, \Lambda)}\right\}}
$$

where $E_{\Lambda}^{\Delta}$ is the interval $[e \cdot N(\Lambda), e \cdot N(\Lambda)+\Delta \cdot N(\Lambda)]$. 
We can extend this functional to all $n$ such that $0 \leqq n \leqq 1$ in the third index.

We begin with the following

Theorem 7. Let $T_{3} \subset \mathfrak{B} \times E_{\Phi} \times[0,1]$ be the set of the $(\Phi, e, n)$ such that the graph of $s^{\Phi}$ has a unique tangent functional in the dual of $\mathfrak{B}$ at $\Phi$, then for $(\Phi, e, n) \in T_{3}$ the equilibrium state $\varrho_{\Phi, e, n}$ is determined by the tangent functional $\alpha_{\Phi, e, n}$ at $\Phi$ and is ergodic. Further we have for $(\Phi, e, n) \in T_{3}$ and for all $\Psi \in \mathfrak{B}$

$$
\varrho_{\Phi, e, n}\left(A_{\Psi}\right)=\alpha_{\Phi, e, n}(\Psi)=\lim _{\Delta \rightarrow 0} \sup _{\Lambda(\Delta) \rightarrow \infty} \alpha_{\Phi, e, n, \Lambda}^{\Delta}(\Psi) .
$$

Furthermore each tangent functional to the graph of $s$ at $(\Phi, e, n)$ determines an equilibrium state $\varrho_{\Phi, e, n} \in E \cap L_{Z^{v}}^{\perp}$ through the relation

$$
\alpha_{\Phi, e, n}(\Psi)=\varrho_{\Phi, e, n}\left(A_{\Psi}\right) \quad \text { for all } \Psi \in \mathfrak{B},
$$

The proof of this Theorem uses one Lemma. For that let $\Phi \in \mathfrak{B}, \Lambda \subset Z^{v}$, $\Lambda$ finite and $e \in E_{\Phi}, 0 \leqq N \leqq N(\Lambda)$ we define for a given $\Delta \in \mathfrak{R}^{+}$

$$
\gamma_{\Phi, e, \frac{N}{N(\Lambda)}, \Lambda}^{\Delta}(\Psi)=\frac{1}{N(\Lambda)} \frac{\operatorname{Tr}_{\mathscr{H}(\Lambda)}\left\{P^{N}(\Lambda) \sum_{\lambda_{i} \in E_{\Lambda}^{A}} E_{\lambda_{i}(\Phi, \Lambda)} e^{-U_{\Phi}(\Lambda)} U_{\Psi}(\Lambda)\right\}}{\operatorname{Tr}_{\mathscr{H}(\Lambda)}\left\{P^{N}(\Lambda) \sum_{\lambda_{i} \in E_{\Lambda}^{A}} E_{\lambda_{i}(\Phi, \Lambda)} e^{-U_{\Phi}(\Lambda)}\right\}} .
$$

We extend this functional to all $n$ such that $0 \geqq n \geqq 1$ in the third index. Now we define also the function

$$
t_{\Delta, \Lambda}^{\Phi}\left(e, \frac{N}{N(\Lambda)}\right)=\frac{1}{N(\Lambda)} \log \operatorname{Tr}_{\mathscr{H}(\Lambda)}\left\{P^{N}(\Lambda) \sum_{\lambda_{i} \in E_{\Lambda}^{\Lambda}} E_{\lambda_{i}(\Phi, \Lambda)} e^{-U_{\Phi}(\Lambda)}\right\}
$$

and we extend this functional to all $n$ such that $0 \geqq n \geqq 1$ in the second variable. With these definitions we have the following

Lemma. $\gamma_{\Phi, e, n, \Lambda}^{4}(\Psi)$ is the tangent functional at $\Phi$ to the function $t_{\Delta, \Lambda}^{\Phi}(e, n)$ for all $\Phi \in \mathfrak{B}$.

Proof. We have

$$
\begin{aligned}
\operatorname{Tr}_{\mathscr{H}(\Lambda)} & \left\{\left.P^{N}(\Lambda) \frac{d}{d \lambda} E_{\Phi+\lambda \Psi}^{\Delta}(\Lambda)\right|_{\lambda=0} e^{-U_{\Phi}(\Lambda)}\right\} \\
& =\frac{1}{2 \pi i} \operatorname{Tr}_{\mathscr{H}(\Lambda)}\left\{P^{N}(\Lambda) \int_{C_{\Lambda}^{A}} \frac{1}{Z-U_{\Phi}(\Lambda)} U_{\Psi}(\Lambda) \frac{1}{Z-U_{\Phi}(\Lambda)} d Z e^{-U_{\Phi}(\Lambda)}\right\} \\
& =0
\end{aligned}
$$

where we have noted $E_{\Phi}^{\Delta}(\Lambda)=\sum_{\lambda_{i}(\Phi, \Lambda) \in E \Delta} E_{\lambda_{i}(\Phi, \Lambda)}$ and $C_{\Lambda}^{\Delta}$ is a contour in $C$ such that $E_{\Lambda}^{\Delta}$ is within and for small $\lambda$ there are no eigenvalues of $U_{\Phi+\lambda \Psi}(\Lambda)$ $=U_{\Phi}(\Lambda)+\lambda U_{\Psi}(\Lambda)$ on $C_{\Lambda}^{4}$. 
The last equality follows by commutativity of $e^{-U_{\Phi}(\Lambda)}$ and $\frac{1}{Z-U_{\Phi}(\Lambda)}$ and cyclicity of $\mathrm{Tr}$.

The Lemma results from the definition of $t_{\Delta, \Lambda}^{\Phi}(e, n)$ and analogy with Theorem 5 or 3 .

Also we recall that the properties of the microcanonical entropy implies [5], that:

where

$$
s^{\Phi}(e, n)=\lim _{\Lambda \rightarrow \infty} s_{\Delta, \Lambda}^{\Phi}(e, n) \quad \text { for all } \Delta
$$

$$
s_{\Delta, \Lambda}^{\Phi}\left(e, \frac{N}{N(\Lambda)}\right)=\frac{1}{N(\Lambda)}\left[\Omega_{\Lambda}^{\Phi}(N(\Lambda)(e+\Delta), N)-\Omega_{\Lambda}^{\Phi}(e \cdot N(\Lambda), N)\right] .
$$

Now to prove Theorem 7, we have

$$
s_{\Delta, \Lambda}^{\Phi}(e, n)-e \geqq t_{\Delta, \Lambda}^{\Phi}(e, n) \geqq s_{\Delta, \Lambda}^{\Phi}(e, n)-e+\Delta
$$

because

$$
\begin{aligned}
\operatorname{Tr}\left\{P^{N}(\Lambda) E_{\Phi}^{\Delta}(\Lambda)\right\} e^{-[e N(\Lambda)+\Delta N(\Delta)]} & \leqq \operatorname{Tr}_{\mathscr{H}(\Lambda)}\left\{P^{N}(\Lambda) E_{\Phi}^{\Delta}(\Lambda) e^{-U_{\Phi}(\Lambda)}\right\} \\
& \left.\leqq \operatorname{Tr}_{\mathscr{H}(\Lambda)}\left\{P^{N}(\Lambda) E_{\Phi}^{\Delta}(\Lambda)\right\} e^{-e \cdot N(\Lambda)}\right\} .
\end{aligned}
$$

Furthermore we note that a tangent functional $\gamma \in \mathfrak{B}$, to $s^{\Phi}(e, n)$ also is a tangent functional to $s^{\Phi}(e, n)-e$.

Then

$$
\begin{aligned}
s^{\Phi+\Psi}(e, n)-e & \geqq s^{\Phi}(e, n)-e-\left(\gamma_{\Phi, e, n, \Lambda}^{4}(\Psi)+\Delta\right)-\varepsilon \\
& \geqq s^{\Phi}(e, n)-e-\left(e^{\Delta} \alpha_{\Phi, e, \Lambda}^{\Delta}(\Psi)+\Delta\right)-\varepsilon \\
& \geqq s^{\Phi}(e, n)-e-\alpha_{\Phi, e, n}(\Psi)-2 \varepsilon
\end{aligned}
$$

which concludes the proof.

Acknowledgments. The author thanks Prof. D. W. Robinson for his contribution to the proof of Theorem 2 and Lemma of 7. He also wants to thank Prof. O. E. Lanford III for critical reading of the manuscript and both for helpful discussions.

\section{References}

1. Lima, R.: Equivalence of ensembles in quantum lattice systems. Ann. Inst. Henri Poincaré 1, 61-68 (1971).

2. Ruelle, D.: Commun. math. Phys. 5, 324-329 (1967).

Gallavotti, G., Miracle-Sole, S.: Commun. math. Phys. 5, 317-323 (1967).

3. Robinson, D. W.: Commun. math. Phys. 6, 151-160 (1967).

4. Lanford, O.E., Robinson, D. W.: Commun. math. Phys. 9, 327-338 (1968).

5. Griffiths, R. B.: J. Math. Phys. 6, 1447-1461 (1965). 